\title{
ORGANIZATIONAL SEMIOTICS: A NORMATIVE AGENT-BASED APPROACH TO VE MODELLING
}

\author{
Joaquim Filipe \\ José Cordeiro \\ School of Technology of Setúbal \\ Polytechnic Institute of Setúbal \\ PORTUGAL \\ (jfilipe, jcordeir)@est.ips.pt
}

\begin{abstract}
Organizational Semiotics is the branch of Semiotics that studies the application of the Theory of Signs in organizational contexts. Organizations are based upon co-coordinated patterns of behavior, including social norms. To achieve the required efficacy, an organization may use both norms and communication to co-ordinate the different agents involved in organizational behavior. The formalization of normative agent behavior can be done using Deontic Agency Logic. The implementation can be done using agent-oriented programming. We propose to combine Ontology Charts, Norm Analysis, Deontic Agency Logic and Agent-Oriented Programming in order to create normative agentbased models of the structure and the dynamics of Virtual Enterprises (VE). This approach seems to be more resistant to organizational change than pure technology-oriented approaches.
\end{abstract}

\section{INTRODUCTION}

Organizational Semiotics is the branch of Semiotics that studies the application of the Theory of Signs in organizational contexts. The underlying philosophical perspective of this approach to organizational modeling is essentially social constructivism focused on resolving semantic problems. Semantic patterns and social norms are some of the concepts that are represented using organizational semiotics methods. The formalization of these concepts can be made using a modal logic that combines the normative ought-to-be operator with an agency operator. The result is a deontic agency logic, which can be partially implemented using an agent-oriented programming paradigm.

Organizations are based upon co-coordinated patterns of behavior. To achieve the required efficacy, an organization may use both norms and communication to co-ordinate the different agents involved in organizational behavior. An efficient organization needs also to be partially automated. However, it is difficult to ensure that all the concerned parties understand the domain of discourse and agree on the formal representation that supports the technical part of an organizational information system. This problem highlights the importance of using a social constructivist perspective in the design of organizational information systems, which is often completely overlooked by traditional software engineering methodologies. A solution for this problem has been proposed by Stamper (1996) in the form of 
Ontology Charts that describe the semantics of a domain in a formal yet understandable way for all stakeholders to agree on the meaning of the terms and relationships used in the organizational information system.

Our perspective is that a social view on system requirements, and the use of formal models where the responsibility of agents is explicit, is at least as fundamental for the success of an organizational information system as the adequate software implementation; therefore it seems to us that the combination of Ontology Charts and Norm Analysis with Deontic Agency logic and Agent-oriented programming could be the key to a new methodology for developing successful organizational information systems.

\section{ORGANIZATIONAL SEMIOTICS}

\subsection{Sign: an Agentive Foundation for Virtual Enterprise Ontology}

One of the concerns of semiotics is precisely to say whether and how we use signs to refer to something. However, there is another question that is: what induces us to produce signs? This question cannot be avoided ontogenetically, as we experience it in our day-to-day experience. Structural semiotics has however never addressed it: the various languages are considered as systems that are already constituted (and synchronically analysable) the moment speakers express themselves.

The first researcher who made this question the very foundation of his theory semiotic, cognitive and metaphysical - was Peirce (see his collected works: 19311958). The reason and process by which we produce and understand signs is from where the problem of being arises, i.e. it makes it necessary to clarify our ontology. In this respect we agree with Heidegger (1962): the problem of being is posed only to those thrown into being-there, into the Dasein, of which our disposition both to notice that something is there and to talk about it is a part. Heidegger postulates that knowledge of the world can only be acquired by being there, in an active role. Peirce also advocates the activity principle as foundational: he suggested that the recognition of things in the world is made by a process that he designated as semiosis. This process is actually an interpretation process by which someone creates in his mind a representation of some object in the world.

The classical definition of a sign "aliquid stat pro aliquo" was modified by Peirce, who expanded it into " $a$ sign is something which stands to somebody for something in some respect or capacity". Peirce's definition emphasizes that a sign has at least three aspects: Some physical representation, something to which this refers or alludes and somebody able to interpret this relationship and form a concept. A concept, as much a percept (see below), is a mental notion that is formed in an interpreter's mind. These elements constitute what is usually called the representational triangle, as shown in figure 1. 


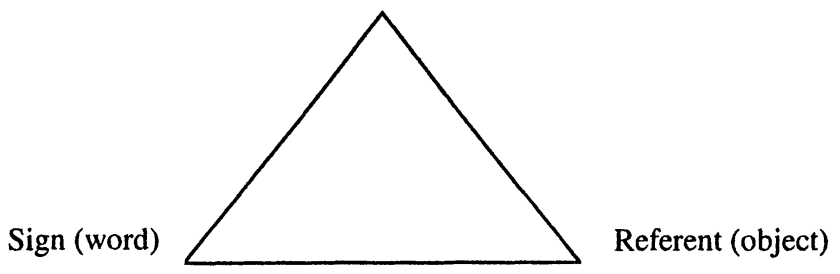

Figure 1 - Peirce's representational triangle

\subsection{Information and Signs: the Semiotics Ladder}

Traditionally, semiotics has been studied in three separate but strongly interconnected dimensions, reflecting the philosophical roots of the subject, which Morris (1946) designated as syntactics, semantics, and pragmatics.

Stamper (1996) pointed out the importance of studying other technical aspects of signs, including the physics of signs, concerning the media in which signs are embodied and the hardware involved in their usage, and the empirics of signs, which studies statistical properties of sets of signs corresponding to the field of what is commonly designated as 'information theory' (Shannon and Weaver, 1949). More importantly, Stamper also pointed out the need to account for the social dimension of signs, and study the social aspects they are involved in, including the creation, modification or termination of commitments and other social relationships. Stamper thus expanded semiotics dimensions from three to six (figure 2).

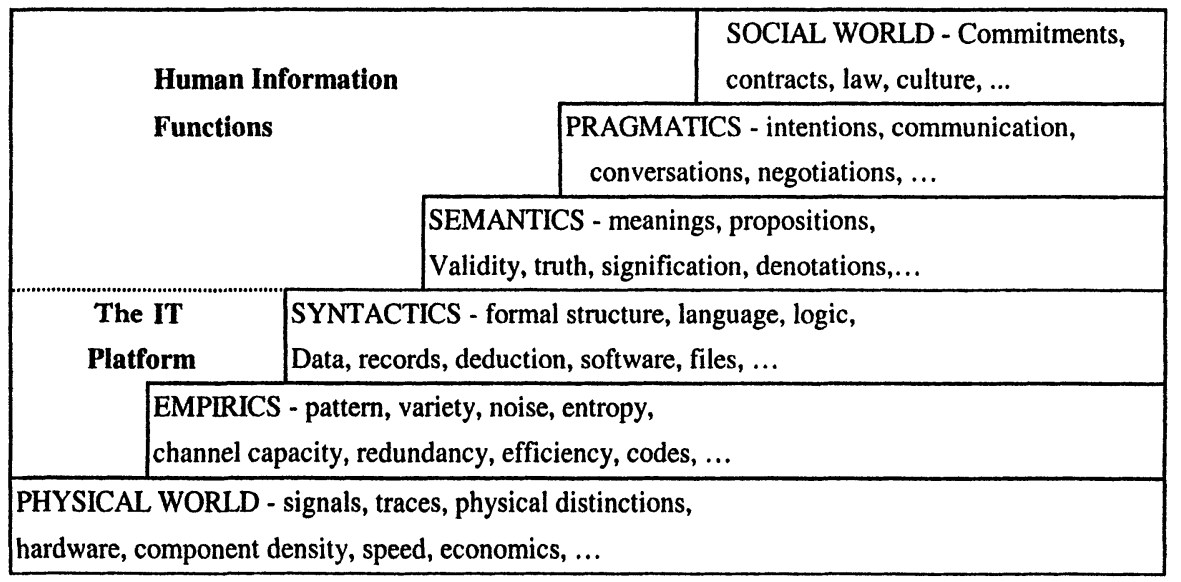

Figure 2 - The Semiotics ladder (Stamper, 1973) 


\subsection{Agents + Affordances = Norms: the Basis for VE Coordination}

The theory of affordances, proposed by Gibson (1966) in the field of perception, in the psychological domain, claims that an affordance is an invariant that an agent identifies in an information-overloaded environment. In this theory objects are no longer thought about as things that stand by themselves with a set of properties, but as a repertoire of behaviors that are warranted to an agent that uses them. The entityrelationship-attribute confusion vanishes from this perspective (Stamper, 1996). Furthermore, the notion of invariant that Gibson introduced to account for a physical percept was extended by Stamper to include the invariants that are perceived in a social world. The invariants in a social world are social norms.

Combining the constructivist stance with Gibson's theory of affordances and Heidegger's Dasein perspective, we obtain an ontology that views organizations as composed by a society of autonomous and responsible agents that, through a continuous interpretation process, co-ordinate their activity using a set of social norms and communication links, based on the following ontological assumptions (Stamper, 1996):

- There is no reality without an agent, and

- The agent constructs reality through its actions

From these assumptions, it is derived that a norm is a well-formed formula with the following structure:

$$
<\text { norm> : : = <agent-term> <action-term> }
$$

This ties every item of knowledge to an agent, who is, in a sense, responsible for it. Instead of an objective truth, there is a concept of truth that corresponds to the set of agents' beliefs. Agents are also responsible for the consequences of the beliefs they choose to adopt.

In a business environment, norms are the coordination instrument that establishes the expected behavior of the organization, both in a prescriptive and proscriptive way, whereas communication is used as a complement to norms, to address situations that cannot be handled exclusively by norms. An important class of situations is what in software engineering is designated as exceptions, which Heidegger (1962) would refer to as break downs, i.e. situations in which objects and properties that were taken for granted (ready-to-hand in Heidegger's terminology), become present-at-hand, i.e. their existence and understanding becomes relevant to achieving our goal.

With respect to normative knowledge representation, it is noted in (Liu and Dix, 1997) that any behavioral norm can be formally specified using a standard generic pattern, based on the use of deontic operators mediating between agent and action, which can be seen as a specialization of ECA (Event-Condition-Action) rules in temporal databases. The deontic operator is used to express the normative character of these rules. Below is a BNF description:

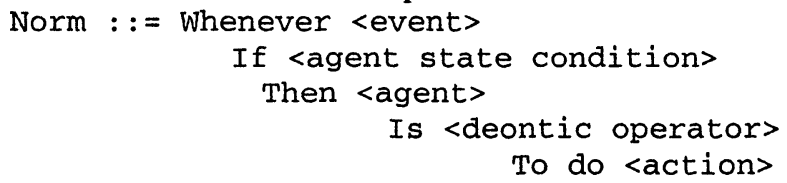

Deontic operator : := obliged | permitted | forbidden 


\subsection{Ontological dependencies: existential, not taxonomic}

Using this ontology, it is possible to specify that certain repertoires of behavior (affordances) depend on the existence of other repertoires of behavior.

Ontology charts emphasize ontological dependencies between affordances. For example, an agent $A$ who is experiencing some invariant $x$, such as being an employee, can go on to experience some other kinds of behavior such as being fired. This dependency of the existence of one kind of behavior upon another is what is called ontological dependency. Figure 3 shows an example of an ontology chart, which depicts an organizational framework, in the Polytechnic Institute of Setúbal, related to book acquisitions. In this diagram we have followed some conventions (see below ${ }^{1}$; those in squared brackets are optional):

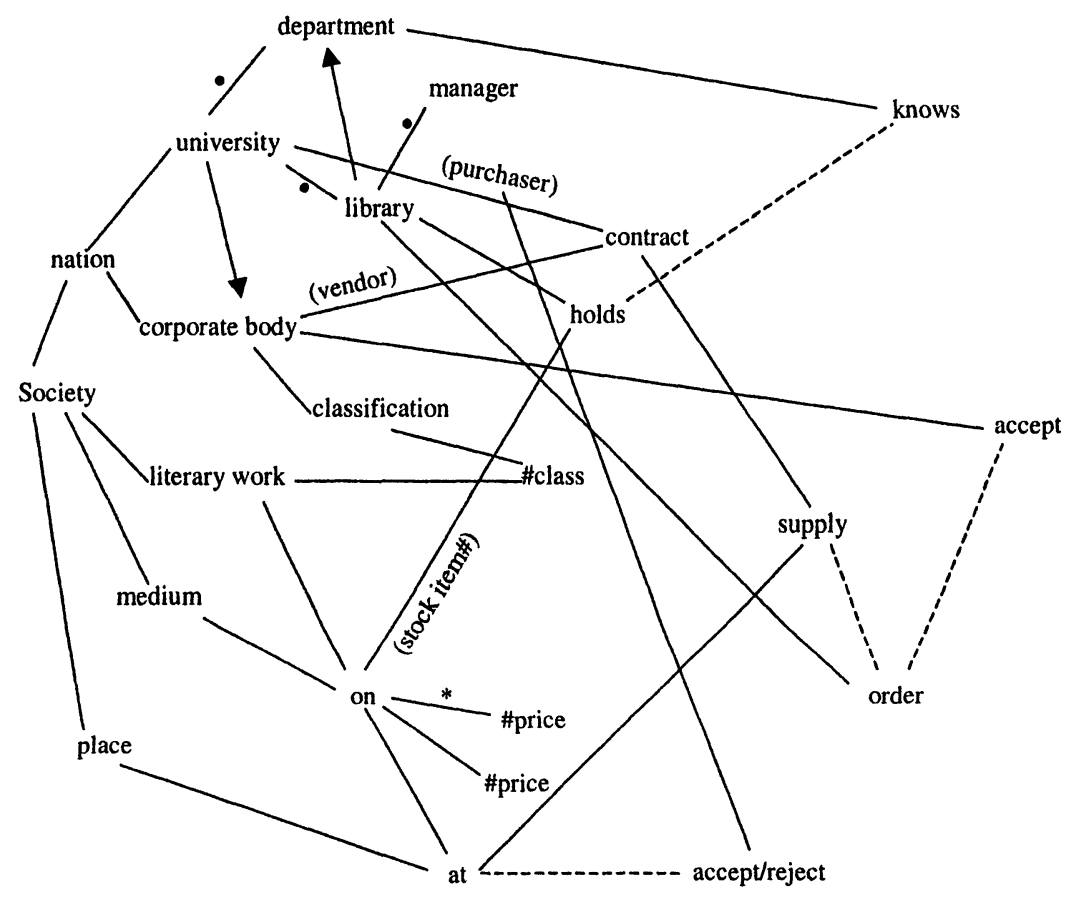

Figure 3 - Ontology Chart of the book acquisition process.

\section{' ONTOLOGY CHART CONVENTIONS:}

a) letter case: lower case for universals; upper case capital for particulars.

b) arcs: broken dependency arc for semiological antecedent; dotted arc for a universal / particular relationship (see an example of the second in figure 9.3).

c) arc labels: a dot labels an arc of a part-whole antecedent; an * labels an arc when the antecedent is the universal rather than a particular - notice that there are prices for the literary work on a certain medium, usually supplied by the publisher, and prices of particular copies of the work.

d) special signs: \# as prefix for a determiner; [one can add a \# suffix for an affordance with individual, identifiable particulars]; arrow points from specific to generic.

e) constraint: maximum of two antecedents which can have role (relative) names.

f) other conventions: role names for antecedents in parentheses: e.g. (purchaser); [without role names they can be labelled $a$ and b]; [affordances that are human agents, individual or collective, may be underlined]; [roles (not relatives) occupied by agents, such as jobs, offices, etc., may be in underlined italics]. 
This graphical representation shows the existing entities and their ontological dependencies but not the starting and finishing times of each one of them. The dynamics of the organization, established by these start and finish events may be determined either by norms or by agents taking responsibility and exercising their choices.

This existential-dependency link introduces the logical property of ontological dependency that is not captured by traditional software engineering methods or even artificial intelligence knowledge representation methods, which tend to focus on taxonomic dependencies, using the so called is-a links. Is-a links are essentially class-subclass or class-instance relationships across which more specific entities inherit properties from the more general ones; this model that has some problems, especially where multiple inheritance is concerned.

\section{AGENCY FORMALIZED}

Organized activity and co-ordination are very close terms, therefore to model organizations it becomes very important to consider how agents represent and use normative knowledge. In a seminal work, von Wright (1951) demonstrated that the important organizational normative notion of 'obligation' cannot be completely understood without accounting for its use in agentive sentences, because typical obligations are 'obligations-to-do' something. Therefore, we will approach these topics in this chapter, discussing both the formal representation of obligations (deontic logic) and actions (action logic).

\subsection{The stit Theory}

Belnap (1991), inspired in the work done by Austin (1962) on Speech Act Theory, suggested a new class of sentences, whose fundamental syntactic and semantic structures are specifically designed to handle sentences describing an action performed by an agent making a choice among alternatives, which he called 'agentives'. The agentive form is composed of two parts: the agent that performs the action, and a complement that describes the consequence of the action. The canonical form proposed to represent this kind of sentences is $[\alpha$ stit $: Q]$, reading " $\alpha$ sees to it that Q", where $\alpha$ designates an agent and 'Q' a complement. For example: [Joe stit: the TV is turned on]. This 2-place operator is quite similar to the agent-affordance relationship that constitutes the norm wff $(<$ norm $>::=<$ agentterm $><$ action-term $>$ ), one of the cornerstones of organizational semiotics.

There are however semantic differences: Whilst the semiotics relationship defines what an agent is afforded to do (can do), the stit operator defines what the agent does. As we describe below, the stit operator can be combined with other operators such as the obligation operator to define what the agent ought-to-do yielding a modal logical representation of the semiotic normative relationship.

\subsection{Deontic Agency Logic}

Deontic logic is the logic of obligations (von Wright, 1951). In this logic it is possible to express that a proposition ought-to-be the case, i.e. $O$ (wff), where wff is 
a well-formed-formula. This modal logic can be given a possible-worlds semantics. In that case, the world where wff $O$ (wff) $\square$ wff is called the ideal world (where all the obligations are fulfilled. Other worlds are sub-ideal but it is still possible to use deontic logic to reason in these sub-ideal worlds. Aqvist (1984) presented a standard system of deontic logic, or SDL as a normal modal logic:

(KD1) $O(\varphi \supset \psi) \supset(O \varphi \supset O \psi)$

The so-called K-axiom

(KD2) $O \varphi \supset \varphi$

The so-called D-axiom

(KD3) $P \varphi \equiv \neg O \neg \varphi$

Permission is dual of obligation

(KD4) $F \varphi \equiv \neg P \varphi$

Forbidden is the same as not permitted

(KD5) Modus Ponens: $(\varphi, \varphi \supset \psi) \square \psi$

(KD6) O-necessitation: $\varphi \square O \varphi$

Standard deontic logic has some practical problems, being plagued with paradoxes (Meyer, 1988). Furthermore, the $O$ operator is ambiguous (ought-to-be vs. ought-to-do). We suggest that these problems can be solved by making the convention that $O$ is read as ought-to-be and making the combination of the $O$ modal operator with the stit agency modal operator, yielding statements such as:

$O[\alpha$ stit: $Q]$ i.e. $\alpha$ ought to see to it that $Q$

$P[\alpha$ stit: $Q]$, i.e. $\alpha$ is permitted to see to it that $\mathrm{Q}$

$F[\alpha$ stit: $Q]$ i.e. $\alpha$ is forbidden to see to it that $Q$

Although these statements are not agentives (in the technical sense) they are quasi-agentive in the sense that, like an agentive, they have the agent as a recoverable part of its intension.

\subsection{Example of Application of Deontic Agency Logic to a VE Model}

Let us now consider the organization depicted in figure 4, which represents a hierarchy of four agents. The power relationships in this case are the following: $\alpha$ has power over $\beta$ with respect to any service this agent is capable of performing; agent $\beta$ has a similar power over agents $\gamma$ and $\delta$; no other direct power relationships are defined - specifically $\alpha$ has no direct power over agents $\gamma$ and $\delta$.

Suppose that we want to specify that agent $\alpha$ has the responsibility to provide service $\mathrm{S}$. Then $\alpha$ has to fulfill its responsibility by exercising its power over $\beta$ in

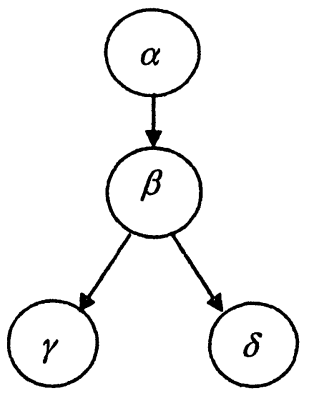

Figure 4: A four agent hierarchy. order that it exercise its power over agents $\gamma$ or $\delta$, both having the capability to stit $\mathrm{S}$. According to the proposed representation, the responsibility of $\alpha$, from the organization perspective, would be the following: $O[\alpha$ stit : $O[\beta$ stit $: O[\gamma$ stit $: S] \vee O[\delta$ stit $: S]]]$

However, from $\alpha$ 's perspective its duty is represented as $O[\alpha$ stit: $O[\beta$ stit: $Q]]$ where $Q=S \vee \exists_{x} O[x$ stit: $S]$, and it is $\beta$ 's duty $O[\beta$ stit $: O[\gamma$ stit $: S] \vee O[\delta$ stit $: S]]$. As we see this 
representation admits that $O[\beta$ stit: $Q]$ is replaced by the equivalent disjunctive statement: $O[\gamma$ stit $: Q] \vee O[\delta$ stit $: Q]$. This is acceptable in the situation where $[\gamma$ stit $: Q] \vee[\delta$ stit $: Q]$ becomes true, meaning that $O[\beta$ stit $: Q]$ has been fulfilled. However, if $\neg([\gamma$ stit $: Q] \vee[\delta$ stit: $Q])$ then agents $\gamma$ and $\delta$ have violated their obligations and in the process they have caused $\beta$ to violate its own obligation. In this case it is debatable whether the equivalence still applies (Santos and Carmo, 1996).

\section{CONCLUSIONS}

The organizational semiotics perspective provides the means to formalize models of virtual organizations, based on ontological dependencies describing the organization structure and social norms governing the dynamics of the organization activity.

The agent concept is central to organizational semiotics, being one of the two components of a wff formal syntax. Ontology charts and norms as described in organizational semiotics are, however, not easy to translate into a computing device, therefore we propose to use deontic agency logic, a combination of deontic logic and agency logic, to create formal models that are better suited to automation, based on the agent-oriented programming paradigm, although deontic agency logic, being a modal (higher-order) logic, presents some computing difficulties; for example, it is not possible to use efficient methods used in first-order logic, such as resolution, to compute the truth value of an expression.

The constructivist approach presented here can, however, guide the analyst to develop better organization models and gives him/her a powerful tool for implementing virtual organizations using a normative agent paradigm.

\section{REFERENCES}

1. Aqvist, L. Deontic Logic. In D. Gabbay and F. Guenther (Eds.), Handbook of Philosophical Logic vol. II, Reidel, Dordrecht, 1984, pp. 605-714.

2. Austin, J. How to Do Things with Words. Oxford University Press: Oxford, England, 1962.

3. Belnap, N. Backwards and Forwards in the Modal Logic of Agency. Philosophy and Phenomenological Research, vol. II, no. 4, 1991, pp 777-807.

4. Gibson, J. The Senses Considered as Perceptual Systems. Houghton Mifflin, Boston, USA, 1966.

5. Heidegger, M. Being and Time. Harper \& Row, New York, USA, 1962.

6. Liu, K. and A. Dix. Norm Governed Agents in CSCW. Proceedings of the 1st International Workshop on Computational Semiotics, Paris, France, 1997.

7. Meyer, J. A Different Approach to Deontic Logic: Deontic Logic Viewed as a Variant of Dynamic Logic. Notre Dame Journal of Formal Logic, 29 (1), 1988, 109-136.

8. Morris, C. Signs, Language and Behaviour. Braziller, New York, 1946.

9. Peirce, C. Collected papers of Ch. S. Peirce (8 vols.), C. Hartshorne and P. Weiss (Eds). Cambridge, Harvard University Press, 1931-1958.

10. Santos F. and J. Carmo. Indirect Action, Influence and Responsibility. In Brown and Carmo (Eds.), Deontic Logic, Agency and Normative Systems, Springer-Verlag, Berlin, 1996.

11. Shannon, C. and W. Weaver. The Mathematical Theory of Communication. MIT Press, Cambridge, MA, 1949.

12. Stamper, R. Information in Business and Administrative Systems. John Wiley \& Sons, 1973.

13. Stamper, R. Signs, Information, Norms and Systems. In Holmqvist et al. (Eds.), Signs of Work, Semiosis and Information Processing in Organizations, Walter de Gruyter, Berlin, New York, 1996.

14. von Wright, G. Deontic Logic. Mind, 60, 1951, pp.1-15. 